\title{
EXTENDED MSM METHOD TO ESTIMATE THE REACTIVITY OF A SUB-CRITICAL CORE DRIVEN BY AN ACCELERATOR BASED NEUTRON SOURCE
}

\author{
A. Billebaud ${ }^{1}$, J.-L. Lecouey ${ }^{2}$, T. Chevret ${ }^{2 *}$, S. Chabod $^{1}$, X. Doligez ${ }^{3}$, A. Kochetkov ${ }^{4}$, \\ A. Krása ${ }^{4}$, F.-R. Lecolley ${ }^{2}$, G. Lehaut ${ }^{2}$, N. Marie $^{2}$, N. Messaoudi ${ }^{4}$, W. Uyttenhove ${ }^{4 * * *}$, \\ G. Vittiglio ${ }^{4}$, J. Wagemans ${ }^{4}$, V. Bécares ${ }^{5}$, D. Villamarin ${ }^{5}$, and F. Mellier ${ }^{6}$ \\ ${ }^{1}$ Laboratoire de Physique Subatomique et de Cosmologie \\ IN2P3(CNRS)-Université Grenoble Alpes, 53 rue des Martyrs 38026 Grenoble Cedex, France \\ ${ }^{2}$ Laboratoire de Physique Corpusculaire de Caen \\ ENSICAEN/Université de Caen/CNRS-IN2P3, 6 bd Maréchal Juin, 14000 Caen, France \\ ${ }^{3}$ Institut de Physique Nucléaire d'Orsay \\ CNRS-IN2P3/Univ. Paris Sud, 91406 Orsay, France \\ ${ }^{4}$ SCK-CEN, Belgian Nuclear Research Centre \\ Boeretang 200, 2400 Mol, Belgium \\ ${ }^{5}$ Nuclear Fission Division, CIEMAT, Madrid, Spain \\ ${ }^{6}$ Commissariat à l'Énergie Atomique et aux Énergies Alternatives, DEN, DER/SPEX, 13108 \\ Saint-Paul-lez-Durance, France \\ billebaud@1psc.in2p3.fr \\ * now at AREVA-NP, Lyon, France, **now at The Binding Energy bvba, Gent, Belgium
}

\begin{abstract}
The Modified Source Multiplication method is used to determine an unknown reactivity level of a reactor from a known one if one has access to the detector counting for both levels when the reactor is fed by a constant neutron source like an Am-Be source. When available, an accelerator driven source, in continuous mode, can be useful as its intensity can be tunable and then adapted to the experimental conditions. However, in that case, the MSM technique must be extended to account for an external source whose intensity, energy and angular distributions can vary from one measurement to another. In this paper, this Modified Multi-Source Multiplication (MMSM) method is applied to measurements done during the FREYA project in the GUINEVERE facility, operated with the GENEPI-3C accelerator providing a mixture of $(\mathrm{D}, \mathrm{T})$ and $(\mathrm{D}, \mathrm{D})$ neutrons. The monitoring of these sources through the detection of the associated charged particles allows the calculation of the MMSM factors and the estimate of the reactivity values. The results are compared in different configurations with the reactivity obtained with an Am-Be source or in dynamic measurements performed with GENEPI-3C. Their excellent agreement shows the possibility of using such accelerator-based neutron sources for MSM measurements when they are correctly monitored. This is of great interest
\end{abstract}


for deep sub-critical level characterization for which detector count rates per source neutrons might be low.

KEYWORDS: ADS, accelerator based neutron source, MSM method, reactivity monitoring

\section{INTRODUCTION}

The GUINEVERE and FREYA projects were partly dedicated to the important issue of reactivity monitoring of a sub-critical reactor such as an Accelerator Driven System (ADS), a reactor concept envisaged for minor actinide transmutation in nuclear waste reduction strategies. To do so, substantial experimental programs were carried out at SCK-CEN (Mol, Belgium) from 2011 to 2016 at the VENUS-F reactor coupled to an external $(\mathrm{D}, \mathrm{T})$ neutron source driven by the GENEPI-3C deuteron accelerator [1], with a titanium tritium target located at the reactor core center. These experiments aimed primarily at testing the validity, accuracy and robustness of reactivity monitoring technique [2] which could be applied during short beam interruptions while the reactor is being operated. Various fast sub-critical cores, with $\mathrm{k}_{\text {eff }}$ ranging from $\sim-18 \$$ to $\sim-4 \$$ were investigated. In order to assess the validity of the reactivity results given by the "beam interruption" techniques, some reference reactivity values were needed for the studied cores to be compared with. They were obtained using the MSM (Modified Source Multiplication) method [3], a wellestablished static measurement method. It is based on the comparison of detector count rates obtained with a neutron source in two different reactor configurations.

MSM measurements are usually performed by inserting a neutron source in the reactor. For some reactor configurations studied in the FREYA project, it was found advantageous to use the GENEPI-3C (D,T) neutron source in continuous mode instead of a standard Am-Be source. However, in this case, the difficulty lies in the normalization of the measurements, in particular because when using a deuteron beam on a solid target containing tritium, after a while some $\mathrm{D}(\mathrm{d}, \mathrm{n})^{3} \mathrm{He}$ reactions will occur in addition to the $\mathrm{T}(\mathrm{d}, \mathrm{n})^{4} \mathrm{He}$ ones. We then have to cope with two neutron sources located at the same place but with different intensities, space and energy distributions which must be accounted for in the MSM correction factor calculation. This makes the neutron production monitoring particularly crucial, as well as the discrimination between $(\mathrm{D}, \mathrm{T})$ or $(\mathrm{D}, \mathrm{D})$ reaction neutrons. We present in this paper an MSM method in an extended form which can be applied when a complex or multiple source, such as an accelerator driven neutron source, is used. We focused on the GENEPI-3C neutron source monitoring and $(D, T)$ versus (D,D) neutron fraction determination. We compare some MSM reactivity values obtained with the GENEPI-3C neutron source to the ones obtained with a standard Am-Be MSM method or with short beam interruption techniques for some configurations.

\section{PRINCIPLE OF THE MODIFIED MULTI-SOURCE MULTIPLICATION METHOD}

The MSM (Modified Source Multiplication) method is a technique for estimating the unknown reactivity of a subcritical configuration by comparing detector count rates driven by an external neutron source in this configuration with those obtained in another subcritical configuration whose reactivity is known.

Let configuration 0 be the subcritical configuration of known reactivity $\rho_{0}$ and configuration 1 be that of unknown reactivity $\rho_{1}$. Assuming that the neutron external source and the detectors utilized are the same in both configurations, the relationship between $\rho_{0}, \rho_{1}$, and the detector count rates $C_{0}$ and $C_{1}$ in configurations 0 and 1 reads [4]:

$$
\frac{\rho_{1}}{\rho_{0}}=f_{M S M} \times \frac{C_{0}}{C_{1}}
$$

The MSM correction factors $f_{M S M}$ must be calculated using a neutron transport code. It is worth mentioning that the value of the MSM correction factor is expected to depend on the detector location. Indeed, any 
difference in the flux shape between the two configurations will result in position-dependent ratios in the $\mathrm{f}_{\text {MSM }}$ formula.

This classical formulation of the MSM method can be readily extended to the case when the neutron source is different for configurations 0 and 1 due to changes in the $(D, T)$ and $(D, D)$ reaction rates. Indeed $S_{i}$, the neutron source intensity (neutrons/s) in measurement $\mathrm{i}$, can be decomposed as $\mathrm{S}_{\mathrm{i}}=\mathrm{S}_{\mathrm{i}}{ }^{\mathrm{DT}}+\mathrm{S}_{\mathrm{i}}{ }^{\mathrm{DD}}$ (DT neutrons/s + DT neutrons/s). Now, introducing $\tilde{C}$, the detector counts per source neutron (instead of per second), which allows for different source intensities between configurations 0 and 1 , formula (1) becomes:

$$
\frac{\rho_{1}}{\rho_{0}}=f_{\text {MSM }} \times \frac{\tilde{c}_{0}}{\tilde{C}_{1}}
$$

The MSM factor can be calculated using a combination of fundamental mode driven simulations and fixedsource ones using DT-like and DD-like neutron sources. Indeed, decomposing the real subcritical flux in two components, one originating from $(\mathrm{D}, \mathrm{T})$ reactions and the other one from $(\mathrm{D}, \mathrm{D})$ reactions for each reactor configuration, the MSM factor can be rewritten as:

$$
f_{M S M}=\frac{\rho_{1}^{c a l c}}{\rho_{0}^{c a l c}} \times\left(\frac{w_{1}^{D T} \times C_{1}^{D T}+w_{1}^{D D} \times C_{1}^{D D}}{w_{0}^{D T} \times C_{0}^{D T}+w_{0}^{D D} \times C_{0}^{D D}}\right)
$$

where we have introduced, for each configuration i:

- $\quad \rho_{i}^{\text {calc }}$, the calculated reactivity of the model used in the neutron transport code, using an iterative fission neutron distribution (e.g. the KCODE card in MCNP) ;

- the DT and DD source fractions $w_{i}^{D T}=S_{i}^{D T} / S_{i}$ and $w_{i}^{D D}=S_{i}^{D D} / S_{i}$, which must be measured experimentally;

- $C_{i}^{D T}$ and $C_{i}^{D D}$ the calculated detector count rates in reactor configuration i using fixed-source simulations with DT-like source neutrons and DD-like neutron sources alone, respectively.

In this paper, the Monte Carlo simulation code MCNP 5 [5] was employed with the JEFF3.1.1 data libraries [6]. MCNP input files describing the simplified geometry of VENUS-F were created for all the reactor configurations of interest.

\section{MSM MEASUREMENTS AT VENUS-F}

The MSM measurements presented in this work were performed at the VENUS-F reactor, a lead fast reactor which can be coupled to an accelerator based neutron source, GENEPI-3C, designed for the GUINEVERE programme [1]. The reactor consists of a cylindrical vessel $(\sim 160 \mathrm{~cm}$ in diameter) containing a square stainless steel casing which can receive $12 \times 12$ assemblies, positioned in a grid. The casing is surrounded by solid lead as a radial reflector. Top and bottom lead reflectors surround the core axially. The assemblies $\left(8 \times 8 \mathrm{~cm}^{2}\right)$ can be absorbent elements (safety and control rods), solid lead (reflector assemblies), solid lead with holes for detectors, or fuel assemblies. When the reactor is used in a sub-critical configuration, the four central assemblies are removed to insert the final part of the GENEPI-3C accelerator beam line, ended by the Tritium target. The measurements presented in this work were performed in configurations shown in Fig.1. Their main features are:

- fuel assemblies composed of 9 high enriched uranium (30 wt.\%) rodlets, 16 lead bars, composing a square surrounded by 4 lead plates

- 6 safety rods (SR) made of $\mathrm{B}_{4} \mathrm{C}$ with a fuel follower

- 2 control rods (CR) and one Pellet Absorber Rod (so-called the PEAR rod) whose height did not change during these experiments.

All detectors used for these experiments (mostly U5 fission chambers) occupy the experimental slots inside the casing, and also the A1, C1 and C2 slots in the outer reflector (see Fig. 1). 
In the FREYA project the reference reactivity levels were mostly determined using the standard MSM method with an Am-Be source, emitting $2.2 \times 10^{6} \mathrm{n} / \mathrm{s}$ and located in the outer reflector (slot A2, see Fig. 1). But in some low reactivity configurations the MSM measurements were performed with the GENEPI-3C neutron source instead. It presents the advantages of reducing significantly the data recording duration as in the continuous beam mode a beam intensity around $300 \mu \mathrm{A}$ can be easily reached, providing a neutron source intensity three orders of magnitude higher (or even more). Due to its flexibility the source can also be adapted to the required count rate of the detectors, to avoid dead time as far as possible.

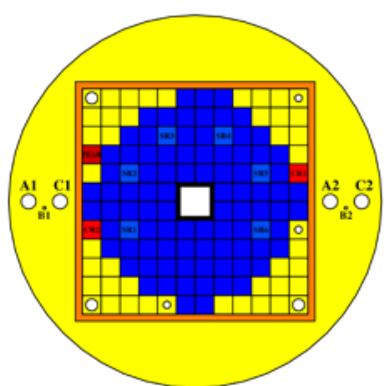

$\mathrm{SC} 1$

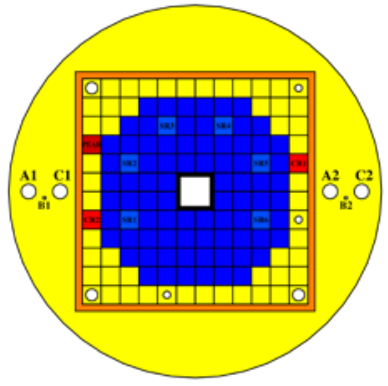

$\mathrm{SC} 2$

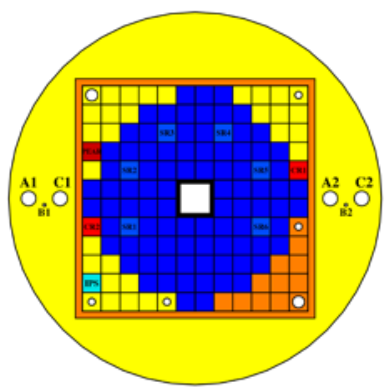

SC1 1SSIPS

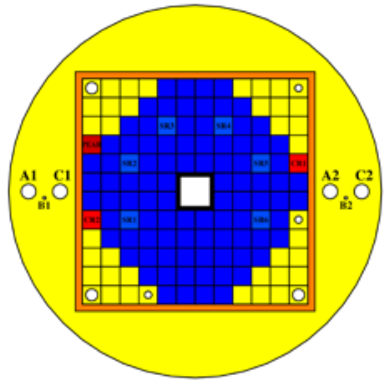

SC3

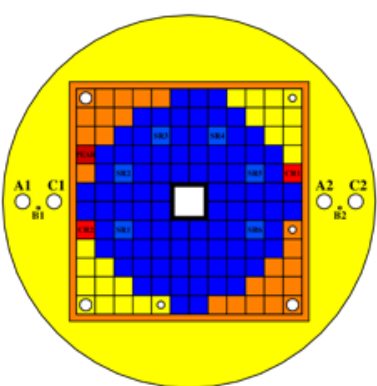

SC1 2SS

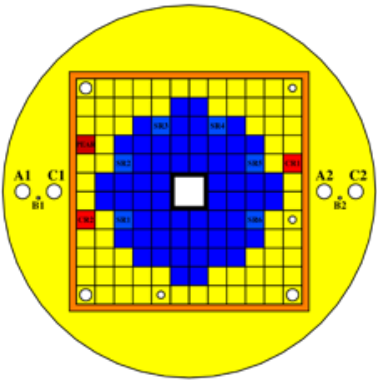

$\mathrm{SC} 4$

Figure 1. : Mid-plane cross-section of the VENUS-F reactor configuration studied. Fuel assemblies are in blue, Control and PEAR Rods in red, lead elements in yellow. Stainless steel is in orange. Experimental slots are in white.

\section{NEUTRON SOURCE MONITORING}

When operating the GENEPI-3C accelerator, the neutron production is monitored by the detection of the fast charged particles, alphas and protons, associated to the $\mathrm{T}(\mathrm{d}, \mathrm{n})^{4} \mathrm{He}$ and $\mathrm{D}(\mathrm{d}, \mathrm{p})^{3} \mathrm{H}$ reactions respectively, this latter being in competition with the $\mathrm{D}(\mathrm{d}, \mathrm{n})^{3} \mathrm{He}$ one. Monitoring these two fast neutron sources is not only necessary to get the relative contribution of the two energy component of the neutron spectrum, but also because the ratio between these two sources may change with time, due to: (i) continuous implantation of deuterium into the target (strongly dependent on the beam history) (ii) aging of the target regarding the tritium contents (iii) beam fluctuations on the target (intensity and position) (iv) time elapsed between the measurements, which can include a target replacement.

This detection is performed by two silicon detectors located around 1 meter upstream from the TiT target, at $178^{\circ}\left(0^{\circ}\right.$ being the beam direction) with the same solid angle for both. Thin Al foils of different thicknesses placed in front of the detectors allow the detection of alphas and protons (API monitor), or 
protons only (PI monitor), in order to make a discrimination between (D,T) and (D,D) neutron productions. Despite the simplicity of the monitoring technique, getting the absolute neutron sources $\left(N_{14 \mathrm{MeV} T o t}\right.$ and $N_{2.5 \mathrm{MeV} \mathrm{Tot}}$ ) for each run is not straightforward. Two aspects have to be addressed: (i) extracting the absolute numbers of alphas $\mathrm{N}_{\alpha \text { API }}$ and protons $\mathrm{N}_{\mathrm{p} \text { PI }}$ seen by API and PI from the experimental energy spectra (ii) determining the factors between the number of charged particles detected and the total number of neutrons (in $4 \pi$ ) produced by the target, $f_{\mathrm{API} \alpha}$ and $\mathrm{f}_{\mathrm{PI}}$, so that we get:

$$
N_{14 \text { MeV Tot }}=\frac{N_{\alpha A P I}}{f_{A P I \alpha}}
$$

and

$$
N_{2.5 \mathrm{MeV} T o t}=\frac{N_{p P I}}{f_{P I}}
$$

\subsection{Analysis of Monitor Spectra}

An example of API $(\alpha+p)$ and PI (p) detector energy spectrum is shown in Fig. 2, both in linear and log scales. Even if the alpha and proton peaks are easy to identify, they are affected by some background counting due to parasitic reactions in silicon, or surrounding material (foils, collimators) and neutron activation. In these figures are also shown the particle peak areas, defined in agreement with their expected shapes, and the subtracted background, approximated to a trapezium. The number of protons is taken from the PI spectrum analysis, and subtracted to the "alphas + protons" peak of the API spectrum, to get the number of detected alphas. In some cases it was necessary to take into account the area of the two-alpha peak due to the simultaneous detection of two alphas, occurring when the neutron production is higher in case of an increased beam intensity or new target.

\subsection{Absolute Neutron Productions}

For a deuteron having an energy $E_{d}$ when interacting with the target, the factors giving the total $(D, T)$ and (D,D) neutron productions from the detection of the alpha and proton particles read as the ratio of the differential cross section to the total cross section at the detection angle and for a defined solid angle:

$$
f_{A P I \alpha}\left(E_{d}\right)=\left(\frac{d \sigma_{\alpha}}{d \Omega}\left(E_{d}\right)\right)_{178^{\circ}} \frac{\Delta \Omega_{A P I}}{\sigma_{\alpha}^{t o t}\left(E_{d}\right)} .
$$

In the case of the $(D, D)$ reaction one has to take into account the neutron production by comparison with the proton production, which reads:

$$
f_{P I p}\left(E_{d}\right)=\left(\frac{d \sigma_{p}}{d \Omega}\left(E_{d}\right)\right)_{178^{\circ}} \frac{\Delta \Omega_{P I}}{\sigma_{p}^{t o t}\left(E_{d}\right)} \frac{\sigma_{p}^{t o t}\left(E_{d}\right)}{\sigma_{n}^{t o t}\left(E_{d}\right)} .
$$

The solid angles read:

$$
\Delta \Omega_{A P I}=\Delta \Omega_{P I} \approx \frac{\pi R_{\text {det }}^{2}}{d_{\text {target-det }}^{2}}
$$

where $R_{d e t}$ is the effective radius of the detectors, defined by a collimator, and $d$ their distance to the target. Although all the quantities of these equations are available, the factors are not so easy to define when the reactions are induced by a continuous spectrum of deuterons, all along their range into the target. 

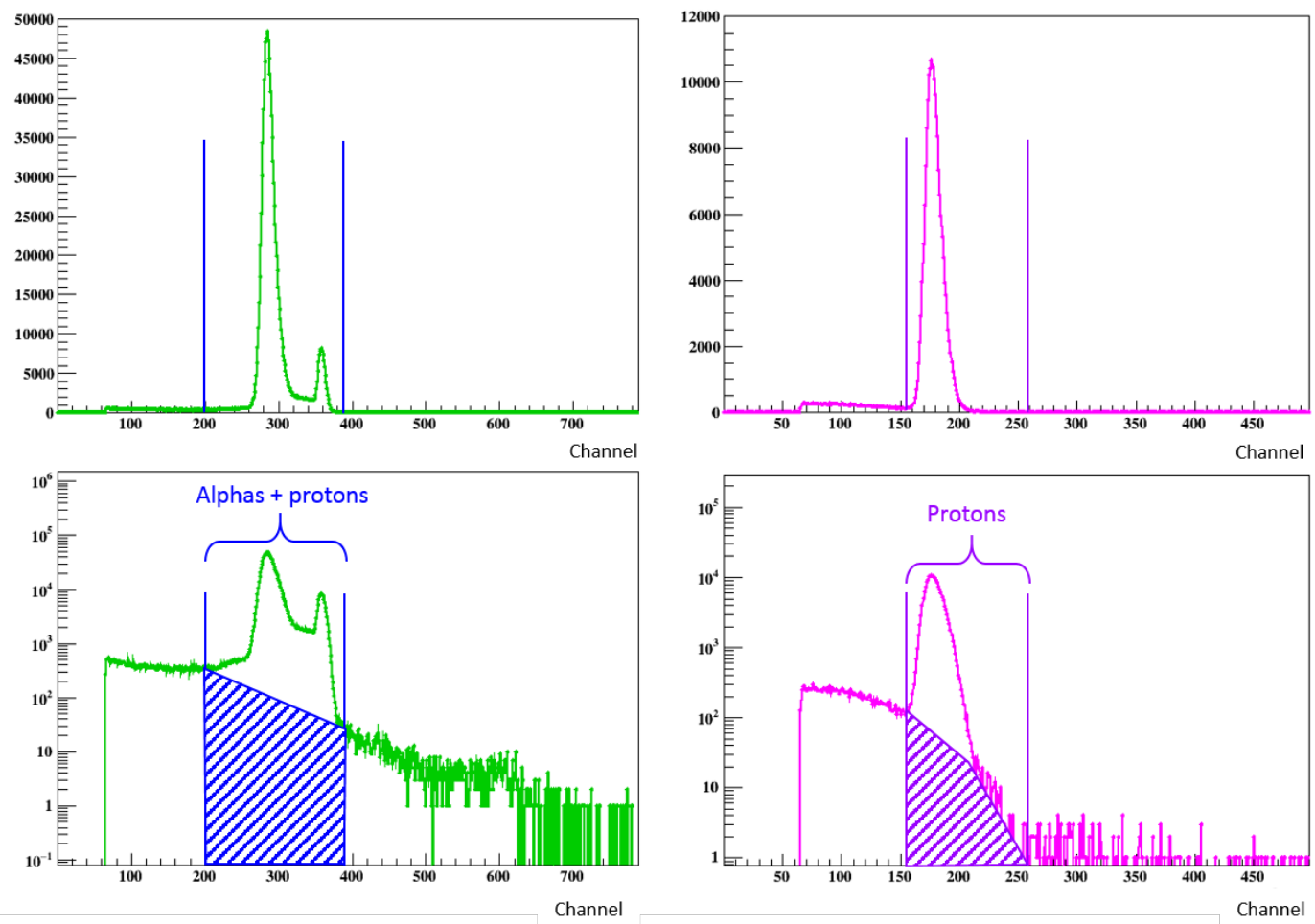

Figure 2. API and PI neutron monitor spectra in both linear and logarithmic scales. Peak area boundaries (between cursors) and subtracted background (hatched) are also shown.

We have to account for their slowing-down from $220 \mathrm{keV}$ to 0 . For each deuteron energy $\mathrm{E}_{\mathrm{di}}$ we have then:

$$
N_{14 M e V}\left(E_{d i}\right)=\frac{N_{\alpha A P I}\left(E_{d i}\right)}{f_{A P I \alpha}\left(E_{d i}\right)}
$$

leading to the total number of produced neutrons:

$$
N_{14 \text { MeV Tot }}=\sum_{i} N_{d i} \frac{N_{\alpha A P I}\left(E_{d i}\right)}{f_{A P I \alpha}\left(E_{d i}\right)}
$$

where $\mathrm{N}_{\mathrm{di}}$ is the number of deuterons of energy $\mathrm{E}_{\mathrm{di}}$. The same formulae can be written for the $2.5 \mathrm{MeV}$ neutron source, replacing $\alpha$ by $\mathrm{p}$, and API by PI. However we have no access to the quantities $N_{\alpha A P I}\left(E_{d i}\right)$ and $N_{p P I}\left(E_{d i}\right)$ experimentally speaking, which are accessible only by simulation. The approach is then done in two steps: the simulation of the total number of alpha (or protons) detected in API (or PI) produced by a $220 \mathrm{keV}$ incident deuteron beam slowing down into the target, and the simulation of the total number of associated neutron production by the same beam in $4 \pi$ :

$$
\begin{gathered}
\left(N_{\alpha A P I}\right)_{s i m}=\sum_{i} N_{d i} N_{\alpha A P I}\left(E_{d i}\right)=\sum_{i} N_{d i}\left(\frac{d \sigma_{\alpha}}{d \Omega}\left(E_{d i}\right)\right)_{178^{\circ}} \Delta \Omega_{A P I} T \\
\left(N_{14 \text { MeV Tot }}\right)_{s i m}=\sum_{i} N_{d i} N_{14 \mathrm{MeV}}\left(E_{d i}\right)=\sum_{i} N_{d i} \sigma_{\alpha}^{t o t}\left(E_{d i}\right) T
\end{gathered}
$$




$$
\begin{gathered}
\left(N_{p P I}\right)_{s i m}=\sum_{i} N_{d i} N_{p P I}\left(E_{d i}\right)=\sum_{i} N_{d i}\left(\frac{d \sigma_{p}}{d \Omega}\left(E_{d i}\right)\right)_{178^{\circ}} \frac{\sigma_{p}^{t o t}\left(E_{d i}\right)}{\sigma_{n}^{t o t}\left(E_{d i}\right)} \Delta \Omega_{P I} T \\
\left(N_{2.5 \text { MeV Tot }}\right)_{s i m}=\sum_{i} N_{d i} N_{2.5 \mathrm{MeV}}\left(E_{d i}\right)=\sum_{i} N_{d i} \sigma_{p}^{t o t}\left(E_{d i}\right) T
\end{gathered}
$$

where $\mathrm{T}$ stands for the number of target atoms ( $\mathrm{T}$ or D), and where $N_{d}=\sum_{i} N_{d i}$ is the total number of simulated deuterons. It leads to factors representative of the experimental conditions:

$$
\begin{gathered}
f_{A P I \alpha}=\frac{\left(N_{\alpha \text { API }}\right)_{s i m}}{\left(N_{14 \text { MeV Tot }}\right)_{s i m}}=\Delta \Omega_{A P I} \frac{\sum_{i} N_{d i}\left(\frac{d \sigma_{\alpha}}{d \Omega}\left(E_{d i}\right)\right)_{178^{\circ}}}{\sum_{i} N_{d i} \sigma_{\alpha}^{t o t}\left(E_{d i}\right)} \\
f_{P I p}=\frac{\left(N_{p P I}\right)_{\text {sim }}}{\left(N_{2.5 \mathrm{MeV} \mathrm{Tot}}\right)_{\text {sim }}}=\Delta \Omega_{P I} \frac{\sum_{i} N_{d i}\left(\frac{d \sigma_{p}}{d \Omega}\left(E_{d i}\right)\right)_{178^{\circ}} \frac{\sigma_{p}^{\text {tot }}\left(E_{d i}\right)}{\sigma_{n}^{t o t}\left(E_{d i}\right)}}{\sum_{i} N_{d i} \sigma_{p}^{\text {tot }}\left(E_{d i}\right)} .
\end{gathered}
$$

It amounts to make the ratios of the differential cross sections obtained by a weighted average over the deuteron energy spectrum, to the total cross sections obtained by the same weighted average.

Practically we model the TiT target as a series of thin layers and calculate the deuteron energy distribution for each of them with SRIM [7], taking $220 \mathrm{keV}$ as the incident ion energy. We use this distribution to build the weighted cross sections (differential or total) of each layer, by summing the contribution of each deuteron energy bin. For the differential cross-section the $178^{\circ}$ angle (lab system) is chosen by making the assumption that the trajectory of the alpha (or proton) to the detector is not affected by its slowing-down on its exit path, which is realistic considering that the energy of the alpha is around $2.6 \mathrm{MeV}(2.5 \mathrm{MeV}$ for the proton), and that scattering is negligible in this range. Finally, the contributions of all layers are summedup and the factors can be calculated, the ratios being auto-normalized since the same energy distribution $\left(\mathrm{N}_{\mathrm{di}}\right)$ is used for two sums (i.e. the energy spectra integrals). Doing so we get:

$$
\begin{gathered}
f_{A P I \alpha}=\Delta \Omega_{A P I} \times 0.0837 \\
f_{P I p}=\Delta \Omega_{P I} \times 0.0943 .
\end{gathered}
$$

\section{RESULTS}

The $\mathrm{N}_{\alpha \text { API }}$ and $\mathrm{N}_{\mathrm{p} \text { PI }}$ quantities and the subsequent contributions of the $(\mathrm{D}, \mathrm{T})$ and $(\mathrm{D}, \mathrm{D})$ neutron sources respectively were determined for each run obtained with the GENEPI-3C source for each VENUS-F configurations. From these quantities the relative contribution of each source can be easily inferred as $\mathrm{w}_{\mathrm{DD}}$ $=\mathrm{N}_{2.5 \mathrm{MeV} \text { Tot }} /\left(\mathrm{N}_{14 \mathrm{MeV} \text { Tot }}+\mathrm{N}_{2.5 \mathrm{MeV} \text { Tot }}\right)$ and $\mathrm{W}_{\mathrm{DT}}=\mathrm{N}_{14 \mathrm{MeV} \text { Tot }} /\left(\mathrm{N}_{14 \mathrm{MeV} \text { Tot }}+\mathrm{N}_{2.5 \mathrm{MeV} \text { Tot }}\right)$. Thanks to these values the MSM factors were calculated for each detector and applied to each configuration measurement, taking the SC1 configuration as the reference reactivity level. The preliminary results of the Modified MultiSource Multiplication method (MMSM), averaged over all detectors, are given in Table I and compared with the results of the standard Am-Be MSM method and/or the Beam Interruption (BI) technique method [2]. The latter consists of analyzing the decrease curve of the neutron population after interruptions of a continuous beam, a method extensively studied in the FREYA project.

An excellent agreement (within $1 \sigma$ ) between MMSM, BI and Am-Be results is found when the complexity of the source is correctly implemented in the MSM method. It shows that the MMSM method can advantageously replace the standard MSM method in case the standard source does not result in enough counting in the detectors. 
Table I.: Relative neutron source contributions and reactivity values obtained with different measurement techniques.

\begin{tabular}{|c|c|c|c|c|c|}
\hline Configuration & $\mathrm{W}_{\mathrm{DD}}$ & $\mathrm{W}_{\mathrm{DT}}$ & $\rho_{\mathrm{Am}-\mathrm{Be}}(\$)$ & $\rho_{\mathrm{BI}}(\$)$ & $\rho_{\mathrm{MMSM}}(\$)$ \\
\hline $\mathrm{SC} 1$ & $4.8 \%$ & $95.2 \%$ & $-5.28 \pm 0.13[7]$ & $-5.24 \pm 0.07$ & - \\
\hline SC1 1 SSIPS & $4.3 \%$ & $95.7 \%$ & - & $-6.05 \pm 0.09$ & $-6.08 \pm 0.17$ \\
\hline SC1 $2 \mathrm{SS}$ & $3.0 \%$ & $97 \%$ & - & $-5.55 \pm 0.06$ & $-5.60 \pm 0.05$ \\
\hline SC2 & $11.5 \%$ & $88.5 \%$ & - & $-8.10 \pm 0.12$ & $-8.15 \pm 0.21$ \\
\hline SC3 & $7.2 \%$ & $92.8 \%$ & - & $-4.11 \pm 0.06$ & $-4.13 \pm 0.11$ \\
\hline SC4 & $13.7 \%$ & $86.3 \%$ & $-17.68 \pm 0.63$ & $-17.43 \pm 0.24$ & $-17.56 \pm 0.46$ \\
\hline
\end{tabular}

\section{CONCLUSIONS}

The MSM method is a technique for estimating the unknown reactivity of a subcritical configuration by comparing detector count rates driven by an external neutron source in this configuration with those obtained in another subcritical configuration. The extension of the MSM method to complex external neutron sources with intensity and/or composition varying between the two subcritical configurations was tested in the VENUS-F reactor coupled to the GENEPI-3C neutron source. Results show that the MMSM method can be successfully used instead of the standard one, which allows one to take advantage of the high, tunable intensity of accelerator driven neutron sources compared to that of standard radio-isotope neutron sources, making possible MSM measurements of deep subcritical levels.

\section{ACKNOWLEDGMENTS}

This work was partially supported by the $6^{\text {th }}$ and $7^{\text {th }}$ Framework Programs of the European Commission (EURATOM) through the EUROTRANS-IP (contract \# FI6W-CT-2005-516520) and FREYA projects (contract \# 269665), and the French PACEN and NEEDS programs of CNRS. The authors want to thank the VENUS reactor and GENEPI-3C accelerator technical teams for their help and support during experiments.

\section{REFERENCES}

1. M. Baylac et al., "The GENEPI-3C accelerator for the GUINEVERE project", Proceeding of the International Topical Meeting on Nuclear Research Applications and Utilization of Accelerators (AccApp09), AIEA, Vienna, Austria, May 4-8 (2009).

2. T. Chevret et al., "Reactivity measurement of the lead fast subcritical VENUS-F reactor using beam interruption experiments", Proceedings of PHYSOR 2014, The Role of Reactor Physics Toward a Sustainable Future, Kyoto, Japan, September 28 - October 3 (2014).

3. J.-L. Lecouey et al.,"Estimate of the reactivity of the VENUS-F sub-critical configuration using a Monte-Carlo MSM method", Annals of Nuclear Energy, 83, pp. 65-75 (2015).

4. P. Blaise, F. Mellier and P. Fougeras, "Application of the Modified Source Multiplication (MSM) Technique to Subcritical Reactivity Worth Measurements in Thermal and Fast Reactor Systems", IEEE Transactions on Nuclear Science, 58(3), pp. 1166-1176 (2011).

5. "MCNP - A General Monte Carlo N-Particle Code, Version 5", LA-ORNL, RSICC LA-UR-03-1987, Los Alamos National Laboratory (2003).

6. W. Haeck and B. Verboomen, "ALEPH-DLG 1.1.0 - Creating Cross Section Libraries for MCNP(X) and ALEPH", Technical Report NEA/JEFF/DOC-1125, OECD/NEA (2006).

7. J. F. Ziegler, "SRIM: The Stopping and Range of Ion in Matter", http://SRIM.org. 